\title{
MEROMORPHIC FUNCTIONS WITH THREE RADIALLY DISTRIBUTED VALUES
}

\author{
BY \\ ALBERT EDREI
}

Introduction. Let $f(z)$ be a meromorphic function of the complex variable

$$
z=r e^{i \theta} \text {. }
$$

Consider the $q$ radii defined by

$$
r e^{i \omega_{1}}, r e^{i \omega_{2}}, \cdots, r e^{i \omega_{q}}
$$$$
(r \geqq 0) \text {, }
$$

where

$$
0 \leqq \omega_{1}<\omega_{2}<\cdots<\omega_{q}<2 \pi \quad(q \geqq 1) .
$$

We say that the roots of the equation

$$
f(z)=a
$$

are distributed on the radii (1) if there exist at most a finite number of roots of the equation (2) which do not lie on the radii (1).

With this definition, the main result of this paper takes the following form.

THEOREM 1. Let $f(z)$ be meromorphic and such that the roots of the three equations

$$
\begin{aligned}
f(z) & =0, \\
f(z) & =\infty, \\
f^{(l)}(z) & =1
\end{aligned}
$$$$
\left(l \geqq 0, f^{(0)} \equiv f\right),
$$

be distributed on the radii (1). Denote by $\delta\left(a, f^{(l)}\right)$ the deficiency of the value $a$, of the function $f^{(l)}$, and assume

$$
\delta(0, f)+\delta\left(1, f^{(l)}\right)+\delta(\infty, f)>0 .
$$

Then the order $\rho$, of $f(z)$, is necessarily finite and

$$
\rho \leqq \beta=\sup \left\{\frac{\pi}{\omega_{2}-\omega_{1}}, \frac{\pi}{\omega_{3}-\omega_{2}}, \cdots, \frac{\pi}{\omega_{q+1}-\omega_{q}}\right\}\left[\omega_{q+1}=2 \pi+\omega_{1}\right] .
$$

In this theorem, it is possible to modify the condition (6) without altering essentially the conclusion. For instance, minor modifications in the proof of Theorem 1 yield the following Theorem 2 (the proof of which will be omitted).

THEOREM 2. Let the assumptions of Theorem 1 be satisfied, with the exception

Presented to the Society, April 24, 1953; received by the editors November 12, 1953. 
of condition (6). Assume, instead, that the roots of one of the three equations (3), (4), (5) have a finite exponent of convergence $\rho_{1}$.

Then the order $\rho$, of $f(z)$, is necessarily finite and either

$$
\rho=\rho_{1} \text {, }
$$

or else

$$
\rho_{1}<\rho \leqq \beta .
$$

As an immediate consequence of Theorem 1, we obtain a

COROLLARY. Let $f(z)$ be an entire function the zeros of which are real. Furthermore, assume that for some integer $l(\geqq 0)$, the roots of

$$
f^{(l)}(z)=1
$$$$
\left[f^{(0)} \equiv f\right]
$$

are all real. Then the order of $f(z)$ is finite and does not exceed one.

If, in the above statement, the word "real" is replaced by the word "positive," the order of $f(z)$ does not exceed $1 / 2$.

The bound (7) for the order cannot be improved, in general. This is clearly seen on examples such as

$$
f(z)=\cos \left(z^{k / 2}\right) \quad(k=1,2,3, \cdots) .
$$

We also note that functions of the form

$$
\Lambda(z)=\sum_{v=1}^{\infty} \frac{\mu_{v}}{z-\sigma_{v}} \quad\left[\mu_{v}>0, \sigma_{v} \text { real, } \sum \mu_{v}<\infty, \sigma_{v} \rightarrow \infty\right]
$$

are always meromorphic, so that, by suitably choosing the sequence $\left\{\sigma_{v}\right\}$, their order can be made equal to any given non-negative number (finite or infinite). On the other hand, if $\operatorname{Im} z \neq 0$,

$$
\operatorname{Im} \Lambda(z) \operatorname{Im} z<0,
$$

so that all real values are distributed on the real axis.

Functions of the form (8) clearly show that, if we assume a radial distribution of the roots of the equations (3), (4), and

$$
f(z)=1 \text {, }
$$

a condition such as (6) [or the corresponding condition of Theorem 2] is essential to the truth of Theorem 1 [or Theorem 2]. A restriction such as (6) is still necessary if we replace, in (9), $f(z)$ by its first derivative. In order to see this, we take

$$
f(z)=G(z) / G^{\prime}(z),
$$

where $G(z)$ is a canonical product, of finite $\operatorname{order} \rho$, the zeros of which are real 
and simple. It is known [2, p. 37] that each derivative of $G(z)$ has, in addition to its real zeros, at most a finite number of complex zeros. Hence the equations (3), (4), and

$$
f^{\prime}(z)=1
$$

have at most a finite number of nonreal roots. On the other hand, $\rho$, which is an arbitrary finite number, is also the order of $f(z)$. Hence Theorem 1 cannot apply to $f(z)$.

The arguments which we use in the proof of Theorem 1 easily yield

THEOREM 3. Let $g(z)$ be an entire function of the form

$$
g(z)=P(z) e^{Q(z)}
$$

where $Q(z)$ is any entire function and $P(z)$ is an entire function of finite order.

Assume that all but a finite number of the roots of the three equations

$$
g(z)=0, \quad g^{\prime}(z)=0, \quad g^{\prime \prime}(z)=0
$$

are real. Then the order, $\rho$, of $Q(z)$ is necessarily finite and does not exceed one.

This theorem obviously contains the following extension of a result of Pólya $[6$, p. 38, footnote 1a] and Saxer [8, p. 206].

Let $g(z)$ be entire and assume that

(i) $g(z)$ and $g^{\prime}(z)$ have no zeros;

(ii) $g^{\prime \prime}(z)$ has at most a finite number of nonreal zeros (and any number finite or infinite of real zeros).

Then $g(z)$ is of one of the two following forms

I. $g(z)=\exp (a z+b)$, where $a$ and $b$ are constants, or

II. $g(z)=\exp \left[c+e^{i\left(\xi_{z}+\eta\right)}\right]$, where $\xi$ and $\eta$ are real constants, and $c$ is any constant.

It might be of interest to mention (without proof) a stronger form of this proposition. If the condition that $g^{\prime}(z)$ has no zeros is replaced by the condition that all the zeros of $g^{\prime}(z) g^{\prime \prime}(z)$ are real, the possible forms of $g(z)$ become more numerous; however, it is still easy to give them explicitly.

In the last section of this paper we briefly sketch an application of our results.

We consider the class $A$ of all entire functions which have only real zeros and real ones. In view of our corollary, we obtain, by very elementary considerations, the following propositions.

A. If $f(z) \in \mathcal{A}$, and if $f(z)$ is not real for some real value of $z$, then it is necessarily of one of the two following forms:

$$
f(z)=\frac{\sin (\xi z+\eta) e^{i\left(\xi z+\eta_{1}\right)}}{\sin \left(\eta-\eta_{1}\right)} \quad\left(\sin \left(\eta-\eta_{1}\right) \neq 0\right),
$$


where $\xi, \eta$, and $\eta_{1}$ are real constants;

$$
f(z)=\frac{\sin (p[\xi z+\eta]) e^{i(p-1)[\xi z+\eta]}}{\sin (\xi z+\eta)},
$$

where $p[\neq 0,1]$ is an integer and $\xi, \eta$ are real constants.

B. Let $f(z)$ be a real entire function belonging to $\mathcal{A}$. Then all the roots of the equation

$$
f(z)=h \quad(\text { real, } 0 \leqq h \leqq 1)
$$

are real.

Using a theorem of Pólya, B easily yields

C. Let $f(z)$ be an entire function. Assume that there exists an unbounded sequence $\left\{h_{v}\right\}_{0=1}^{\infty}$ such that all the roots of the equations

$$
f(z)=h_{v} \quad(v=1,2,3, \cdots),
$$

be real. Then $f(z)$ is a polynomial of degree not greater than two.

We conclude this introduction by observing that Theorems 1 and 2 express a property of the essential singularity of $f(z)$. They remain true if $f(z)$ is only assumed to be meromorphic in the region

$$
D^{*}(\lambda): \lambda \leqq r<+\infty .
$$

In this case, the only roots of (3), (4), and (5) to be taken into account are those which lie in the region $D^{*}$. Our proofs require only minor modifications in order to yield this extended form of the theorems [cf. footnotes 3, 5, and 6].

1. A theorem of Milloux. Our starting point will be the following lemma contained in some more general results of Milloux [4, p. 25].

LEMma. Let the functions

$$
F(w), \alpha_{0}(w), \alpha_{1}(w), \cdots, \alpha_{l}(w)
$$

be regular for $|w|<1$, and such that

(1.1) $F(w) \neq 0 ; \psi(w)=\alpha_{0}(w) F(w)+\alpha_{1}(w) F^{\prime}(w)+\cdots+\alpha_{l}(w) F^{(l)}(w) \neq 1$,

$$
\psi(0) \neq 0, \quad \psi^{\prime}(0) \neq 0 .
$$

Furthermore assume that, as $t \rightarrow 1(0<t<1)$,

$$
m\left(t, \alpha_{j}(w)\right)=O\left(\log \frac{1}{1-t}\right) \quad(j=1,2, \cdots, l) .
$$

Then

$$
m(t, F(w))=O\left(\log \frac{1}{1-t}\right)
$$


The proof $\left({ }^{1}\right)$ may be obtained by a straightforward application of Nevanlinna's methods. Using properties of Nevanlinna's means and Jensen's theorem, we find

$$
\begin{aligned}
m(t, F) & \leqq m(t, \psi)+m\left(t, \frac{F}{\psi}\right) \\
& =m(t, \psi)+m\left(t, \frac{\psi}{F}\right)-N\left(t, \frac{F}{\psi}\right)+\log \left|\frac{F(0)}{\psi(0)}\right| .
\end{aligned}
$$

We next apply Nevanlinna's theorem [5, pp. 141-143] to the function $\psi$ and use (1.5)

$$
\begin{aligned}
m(t, F) & \leqq m\left(t, \frac{\psi}{F}\right)-N\left(t, \frac{F}{\psi}\right)+N\left(t, \frac{1}{\psi}\right)+\log \left|\frac{F(0)}{\psi(0)}\right|+S(t) \\
& =m\left(t, \frac{\psi}{F}\right)+S(t)+\log \left|\frac{F(0)}{\psi(0)}\right|
\end{aligned}
$$

where

$$
S(t)<K+4 \log \frac{1}{t}+6 \log \frac{1}{\tau-t}+8 \log ^{+} m(\tau, \psi) \quad(0<t<\tau<1) ;
$$

the finite constant $\left({ }^{2}\right) K$ depends on the finite parameters

$$
\frac{1}{|\psi(0)|}, \quad \frac{1}{|\psi(0)-1|}, \quad \frac{1}{\left|\psi^{\prime}(0)\right|} .
$$

Known estimates $[10$, p. 12] yield

$$
\begin{aligned}
m\left(t, \frac{\psi}{F}\right)< & \log (l+1)+\sum_{j=0}^{l} m\left(t, \alpha_{j}\right)+\sum_{j=1}^{l} m\left(t, \frac{F^{(j)}}{F}\right) \leqq \sum_{j=0}^{l} m\left(t, \alpha_{j}\right) \\
& +K \log ^{+} m\left(\tau^{*}, F\right)+K \log \frac{1}{\tau^{*}-t} \\
& +K \log ^{+}|\log | F(0)||+K,
\end{aligned}
$$

where $0<t<\tau^{*}<1$, and the constants $K$ depend on $l$ only.

In view of (1.3), (1.6), and (1.7), the lemma follows from well known arguments of Nevanlinna (or alternatively from the lemma used by Milloux $[4$, p. 24]).

2. A conformal transformation. Let $\Delta_{k}$ be the sector defined by

(1) Milloux assumes that the functions $\alpha_{j}$ do not vanish in the unit circle. Our brief sketch of his proof shows that he does not require this assumption.

(2) We use $K$ to denote a constant, depending on one or more parameters, not necessarily the same one each time it occurs. 


$$
r>1
$$

and write

$$
\frac{\pi}{\gamma}=\omega_{k+1}-\omega_{k}
$$

Consider the conformal transformation

$$
w=\frac{u^{\gamma}-u^{-\gamma}-\kappa}{u^{\gamma}-u^{-\gamma}+\kappa}=\phi_{k}^{-1}(u),
$$

where $\kappa$ is a positive parameter and

$$
u=e^{-i \zeta_{k}}, \quad \zeta_{k}=\frac{\omega_{k}+\omega_{k+1}}{2} .
$$

The function

$$
z=e^{i \zeta k} \phi_{k}(w)
$$

maps the circle $|w|<1$ onto the sector $\Delta_{k}$.

Now (2.2) implies

$$
|w|^{2}=\frac{1-\Omega}{1+\Omega}
$$

where

$$
\Omega=\frac{2 \kappa\left(r^{\gamma}-r^{\gamma}\right) \cos \left(\gamma\left[\theta-\zeta_{k}\right]\right)}{r^{2 \gamma}+r^{-2 \gamma}+2+\kappa^{2}-4 \cos ^{2}\left(\gamma\left[\theta-\zeta_{k}\right]\right)} .
$$

Hence

$$
\frac{1}{2}(1-|w|)<\Omega<2(1-|w|),
$$

so that, for sufficiently large values of $r,(2.4)$ yields

$$
\frac{\kappa}{4} r^{-\gamma} \cos \left(\gamma\left[\theta-\zeta_{k}\right]\right)<1-|w|<8 \kappa r^{-\gamma} .
$$

3. Consequences of Milloux's result. Using the mappings and notations introduced in the previous section, we now prove

LEMMA 1. Let $f(z)$ be meromorphic in the region

$$
\mathcal{D}(1): 1<|z|<+\infty,
$$

and assume that those roots of (3), (4), and (5) which are in $\mathcal{D}(1)$ lie on the radii (1). 
Consider the $q$ functions defined by

$$
F_{k}(w)=f\left(e^{i \zeta_{k}} \phi_{k}(w)\right) \quad(k=1,2, \cdots, q) .
$$

Then, as $t \rightarrow 1(0<t<1)$,

$$
\begin{aligned}
m\left(t, F_{k}(w)\right) & =O\left(\log \frac{1}{1-t}\right), \\
m\left(t, \frac{1}{F_{k}(w)}\right) & =O\left(\log \frac{1}{1-t}\right), \\
m\left(t, \frac{1}{f^{(l)}\left(e^{i \zeta_{k} \phi_{k}(w)}\right)-1}\right) & =O\left(\log \frac{1}{1-t}\right) .
\end{aligned}
$$

Proof. We write

$$
F_{k}(w)=F(w),
$$

and omit systematically the subscript $k$. Put

$$
V(w)=\frac{e^{-i \zeta}}{\phi^{\prime}(w)}
$$

then

$$
f^{\prime}\left(e^{i \zeta} \phi(w)\right)=V(w) F^{\prime}(w),
$$

and an obvious induction shows that

$$
f^{(l)}\left(e^{i \zeta} \phi(w)\right)=\sum_{j=1}^{l} \alpha_{j} F^{(j)}(w)
$$

where the coefficients $\alpha_{j}$ are polynomials (with numerical coefficients) in the variables

$$
V, V^{\prime}, V^{\prime \prime}, \cdots
$$

We take

$$
\psi(w)=f^{(l)}\left(e^{i \zeta} \phi(w)\right),
$$

and choose the parameter $\kappa$, in (2.2), so that

$$
\psi(0) \neq 0, \quad \psi^{\prime}(0) \neq 0 .
$$

This is obviously possible except if $f^{(l+1)}(z) \equiv 0$ and, in this case, the lemma is trivial. By assumption,

$$
F(w) \neq 0, \quad F(w) \neq \infty, \quad \psi(w) \neq 1 \quad \text { for }|w|<1,
$$

so that (3.1) follows from Milloux's lemma provided we show that the functions $\alpha_{j}(w)$ satisfy the conditions (1.3). 
Now (2.2) implies

$$
|V(w)|=\left|\frac{d w}{d z}\right|=\frac{\gamma}{2 \kappa}\left|(1-w)^{2}\left[u^{\gamma-1}+u^{-\gamma-1}\right]\right| .
$$

Hence, in view of (2.2) and (2.5), we obtain, if $\gamma>1$ (and $r$ is sufficiently large),

$$
|V(w)|<\frac{4 \gamma}{\kappa}\left(\frac{8 \kappa}{1-|w|}\right)^{(\gamma-1) / \gamma} .
$$

If $\gamma \leqq 1$, the function $V(w)$ remains bounded in the circle $|w|<1$.

Taking

$$
\tau=|w|+\frac{1}{2}(1-|w|),
$$

we obtain (by Cauchy's theorem and (3.6)),

$$
\begin{array}{rr}
\left|V^{(\mu)}(w)\right| \leqq \frac{\mu !}{(\tau-|w|)^{\mu+1}} M(\tau, V(w)) \leqq \frac{\mu !}{(\tau-|w|)^{\mu+1}} \frac{4 \gamma}{\kappa}\left(\frac{8 \kappa}{1-\tau}\right)^{(\gamma-1) / \gamma} & (|w|<\tau<1), \\
V^{(\mu)}(w)=O\left(\left\{\frac{1}{1-|w|}\right\}^{\{(\gamma-1) / \gamma \mid+\mu+1}\right) & \quad(\gamma>1) .
\end{array}
$$

A similar inequality holds for $\gamma \leqq 1$. Taking into account the special form of the functions $\alpha_{j}$, we immediately verify the conditions (1.3). We have thus proved (3.1) and, in view of Jensen's theorem, also (3.2).

Now (3.5) yields

$$
m\left(t, f^{(l)}\left(e^{i \zeta} \phi(w)\right)\right) \leqq m(t, F(w))+\log l+\sum_{j=1}^{l} m\left(t, \alpha_{j}\right)+\sum_{j=1}^{l} m\left(t, \frac{F^{(j)}}{F}\right),
$$

so that

$$
T\left(t, f^{(l)}\left(e^{i \zeta} \phi(w)\right)\right)=O\left(\log \frac{1}{1-t}\right),
$$

by the estimates used in (1.7). This implies (3.3) (by Nevanlinna's first theorem $[5$, p. 129]).

4. Arcs on which the modulus of certain meromorphic functions is large.

Lemma 2. Let $h(z)$ ( $\not \equiv$ constant) be a meromorphic function $\left(^{(3)}\right.$ the poles of

(3) If $h(z)$ is only assumed to be meromorphic in the region defined by $1 \leqq|z|<+\infty$, we may write $h(z)=h_{1}(z)+h_{2}(z)$ where $h_{1}(z)$ is a meromorphic function and $h_{2}(z)$ is regular for $|z| \geqq 1$ and vanishes at infinity. Lemma 2 may be applied to $h_{1}(z)$ and hence remains true for $h(z)$, provided $T(r, h)$ is suitably defined [cf. footnote 5 ]. 
which have a positive deficiency $\delta$. Denote by $\mathfrak{M}(r)$ the set of values of $\theta[0 \leqq \theta<2 \pi]$ for which

$$
\log \left|h\left(r e^{i \theta}\right)\right|>\frac{\delta}{4} T(r, h) .
$$

Given $\epsilon(>0)$, there exists $\sigma_{0}(\epsilon)(>0)$ such that, if $\sigma>\sigma_{0}(\epsilon)$,

$$
\text { measure } \mathfrak{M}(r)>\frac{1}{T^{\epsilon}(r, h)[\log r]^{1+\epsilon}},
$$

for some $r$ belonging to the interval $(\sigma, 2 \sigma)$.

Proof $\left({ }^{4}\right)$. Let $\left\{b_{v}\right\}_{0=1}^{\infty}$ be the sequence of poles of $h(z)$ (we assume that $\left\{\left|b_{v}\right|\right\}_{v-1}^{\infty}$ is nondecreasing and that the multiplicities of the poles have been taken into account by a suitable repetition of the elements).

Let $I_{v}$ denote the interval

$$
\left(\left|b_{v}\right|-\frac{1}{v^{2}},\left|b_{v}\right|+\frac{1}{v^{2}}\right)
$$

of the real axis. If $r>0$ and $r \notin \sum I_{v}$, the Poisson-Jensen formula yields [5, p. 25]

(4.3) $\log ^{+}\left|h\left(r e^{i \theta}\right)\right| \leqq \frac{R+r}{R-r} m(R, h)+n(R)[\log 2 R+2 \log n(R)] \quad(R>r)$, where $n(R)$ denotes the number of poles in the region $|z| \leqq R$.

Putting $R^{\prime}-R=R-r$, we obtain

$$
N\left(R^{\prime}, h\right)-N(R, h)=\int_{R}^{R^{\prime}} \frac{n(t)}{t} d t>\frac{n(R)}{R^{\prime}} \frac{R^{\prime}-r}{2},
$$

so that (4.3) implies

$$
\begin{aligned}
\log ^{+}\left|h\left(r e^{i \theta}\right)\right| \leqq & \frac{4 R^{\prime}}{R^{\prime}-r} T\left(R^{\prime}, h\right) \\
& +\frac{2 R^{\prime} N\left(R^{\prime}, h\right)}{R^{\prime}-r}\left\{\log 2 R^{\prime}+2 \log \left(\frac{2 R^{\prime}}{R^{\prime}-r} N\left(R^{\prime}, h\right)\right)\right\} \\
\leqq & \frac{4 R^{\prime}}{R^{\prime}-r} T\left(R^{\prime}, h\right)\left[\left(1+\frac{3}{2} \log 2\right)\right. \\
& \left.+\frac{1}{2} \log R^{\prime}+\frac{R^{\prime}}{R^{\prime}-r}+\log T\left(R^{\prime}, h\right)\right] .
\end{aligned}
$$

(4) A lemma which has essentially the same structure is to be found in Milloux $[3$, p. 318]. 
As soon as $r$ exceeds some bound $\sigma_{1}$, each of the four terms in the parenthesis $[\cdots]$ of (4.4) exceeds one, so that

$$
[\cdots] \leqq 4\left(1+\frac{3}{2} \log 2\right)\left(\frac{1}{2} \log R^{\prime}\right)\left(\frac{R^{\prime}}{R^{\prime}-r}\right) \log T\left(R^{\prime}, h\right) .
$$

Hence

$$
\log ^{+}\left|h\left(r e^{i \theta}\right)\right| \leqq A\left(\frac{R^{\prime}}{R^{\prime}-r}\right)^{2}\left(\log R^{\prime}\right) T\left(R^{\prime}, h\right) \log T\left(R^{\prime}, h\right),
$$

where $A=$ constant, $r>\sigma_{1}, r \notin \sum I_{v}$. For $r>\sigma_{1}$, the function

$$
V(r)=[T(r, h) \log T(r, h)] \log r
$$

is positive, continuous, and increases to infinity. Hence given $\eta(>0)$, a well known lemma of Borel [1, p. 18] asserts that

$$
V\left(r\left(1+\frac{1}{\log V(r)}\right)\right)<V^{1+\eta}(r)
$$

except possibly for values of $r\left(>\sigma_{1}\right)$ which belong to an exceptional set $\mathcal{E}(\eta)$, of finite logarithmic measure. Taking, in (4.5),

$$
R^{\prime}=r\left(1+\frac{1}{\log V(r)}\right)
$$

we obtain

$$
\log ^{+}\left|h\left(r e^{i \theta}\right)\right| \leqq V^{1+2 \eta}(r)
$$

provided $r$ is sufficiently large and

$$
r \notin \varepsilon(\eta)+\sum I_{v}
$$

As the interval $[\sigma, 2 \sigma]$ is of logarithmic measure $\log 2$, and $\varepsilon(\eta)+\sum I_{v}$ is of finite logarithmic measure, there will exist, as soon as $\sigma$ exceeds a suitable bound, at least one point $r(\sigma)$ of $(\sigma, 2 \sigma)$ such that (4.6) is true. Consider the measurable set (of values of $\theta[0 \leqq \theta<2 \pi]$ )

$$
E=E\left[\log ^{+}\left|h\left(r e^{i \theta}\right)\right|>\frac{1}{2} m(r, h)\right]
$$

obviously

$$
\begin{aligned}
2 \pi m(r, h) & \leqq \int_{E} \log ^{+}\left|h\left(r e^{i \theta}\right)\right| d \theta+2 \pi \frac{m(r, h)}{2}, \\
\pi m(r(\sigma), h) & <V^{1+2 \eta}(r(\sigma)) m E .
\end{aligned}
$$

If $r$ is sufficiently large, 


$$
\begin{aligned}
m(r, h) & >\frac{\delta}{2} T(r, h), \\
\mathfrak{M}(r) & \supset E .
\end{aligned}
$$

Hence (4.7) implies

$$
\frac{(\pi / 2) \delta T(r(\sigma), h)}{T^{1+2 \eta}(r(\sigma), h)[\log T(r(\sigma), h)]^{1+2 \eta} \log ^{1+2 \eta} r}<m \mathfrak{M}(\boldsymbol{r}) ;
$$

taking $\eta=\epsilon / 3$, we obtain (4.2).

\section{Consequences of Lemma 2.}

Lemma 3. Let $h(z)$ be a meromorphic function of order $\rho$ (not necessarily finite). Assume

(i) that the poles of $h(z)$ either lie on the q radii defined by (1) or else belong to the disk $|z| \leqq 1$;

(ii) that the poles of $h(z)$ have a positive deficiency.

Consider the number $\beta$ defined by

$$
\beta=\sup \left\{\frac{\pi}{\omega_{2}-\omega_{1}}, \frac{\pi}{\omega_{3}-\omega_{2}}, \cdots, \frac{\pi}{\omega_{q+1}-\omega_{q}}\right\} \quad\left[\omega_{q+1}=2 \pi+\omega_{1}\right],
$$

the numbers $\zeta_{k}$ and the functions $\phi_{k}$ defined in $\S 2$.

Then

$$
\beta<\rho
$$

implies

$$
\limsup _{t \rightarrow 1} \frac{m\left(t, h\left(e^{i \zeta k} \phi_{k}(w)\right)\right)}{\log \frac{1}{1-t}}=+\infty \quad(0<t<1)
$$

for some integer $k[1 \leqq k \leqq q]$.

Proof. By assumption (5.2), it is possible to choose three positive numbers $\beta_{1}, \beta_{2}, \eta_{1}$ such that

$$
\rho>\beta_{1}>\beta_{2}>\beta+\eta_{1} .
$$

We next choose an unbounded positive sequence $\left\{R_{v}\right\}_{v=1}^{\infty}$, such that

$$
T\left(R_{v}\right)=T\left(R_{v}, h\right) \geqq R_{v}^{\beta_{1}} \quad(v=1,2,3, \cdots) .
$$

Applying Lemma 2, with

$$
\epsilon=\frac{1}{2}\left(1-\frac{\beta_{2}}{\beta_{1}}\right)
$$


we see that, as soon as $R_{n}>\sigma_{0}(\epsilon)$, there exists at least one point $r_{n}$, belonging to the interval $\left(R_{n}, 2 R_{n}\right)$ and such that (4.2) is true for $r=r_{n}$. We may therefore associate with each $r_{n}\left(>\sigma_{0}(\epsilon)\right)$ at least one argument $\theta_{n}$, belonging to one of the $q$ arcs defined by

$$
\omega_{k}+\frac{1}{2 q T^{\epsilon}\left(r_{n}\right) \log { }^{1+\epsilon} r_{n}} \leqq \theta \leqq \omega_{k+1}-\frac{1}{2 q T^{\epsilon}\left(r_{n}\right) \log ^{1+\epsilon} r_{n}},
$$

and such that

$$
\log \left|h\left(r_{n} e^{i \theta_{n}}\right)\right|>\frac{\delta}{4} T\left(r_{n}\right)
$$

We choose among the $q$ sectors $\Delta_{1}, \Delta_{2}, \cdots, \Delta_{q}$, a sector $\Delta_{k}$ which contains an infinity of terms of

$$
r_{1} e^{i \theta_{1}}, r_{2} e^{i \theta_{2}}, \cdots \text {. }
$$

Renumbering if necessary the elements of this sequence, we may assume that all its terms belong to $\Delta_{k}$.

Hence, using the notations of $\$ 2$, we obtain

$$
\begin{aligned}
& \left|\theta_{n}-\zeta_{k}\right|<\frac{\pi}{2 \gamma}-\frac{1}{2 q T^{\epsilon}\left(r_{n}\right) \log ^{1+e} r_{n}}, \\
& \cos \left(\gamma\left[\theta_{n}-\zeta_{k}\right]\right)>\frac{\gamma}{4 T^{\epsilon}\left(r_{n}\right) \log ^{1+\epsilon} r_{n}},
\end{aligned}
$$

provided $n$ is sufficiently large. The function

$$
H_{k}(w)=h\left(e^{i \zeta k} \phi_{k}(w)\right)
$$

is regular in the unit circle; putting

$$
w_{n}=\phi_{k}^{-1}\left(r_{n} e^{i\left(\theta_{n}-\zeta k\right)}\right),
$$

(5.7) becomes

$$
\log \left|H_{k}\left(w_{n}\right)\right|>\frac{\delta}{4} T\left(r_{n}\right) .
$$

In view of (5.9) and the first of the inequalities (2.5), we obtain

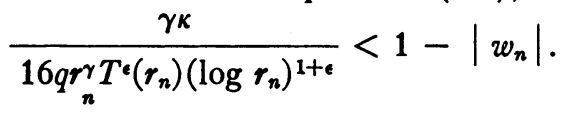

Taking

$$
t_{n}=\left|w_{n}\right|+\frac{1}{2}\left(1-\left|w_{n}\right|\right)
$$


we have $[5$, p. 140$]$

$$
m\left(t_{n}, H_{k}(w)\right) \geqq \frac{t_{n}-\left|w_{n}\right|}{t_{n}+\left|w_{n}\right|} \log \left|H_{k}\left(w_{n}\right)\right|,
$$

so that (5.10), (5.11), (5.12), and (5.13) imply

$$
m\left(t_{n}, H_{k}(w)\right)>\frac{\gamma \delta \kappa}{256 q} \frac{T^{1-\epsilon}\left(r_{n}\right)}{r_{n}^{\gamma} \log ^{1+\epsilon} r_{n}}
$$

If the lemma were not true, we would have

$$
m\left(t, H_{k}(w)\right)=O\left(\log \frac{1}{1-t}\right) .
$$

Combining (5.14) and (5.15), and using (5.11) and (5.12), we obtain

$$
K \frac{T^{1-\epsilon}\left(r_{n}\right)}{r_{n}^{\gamma} \log ^{1+\epsilon} r_{n}}<K+K \log \left(T^{\epsilon}\left(r_{n}\right) r_{n}^{\gamma} \log ^{1+\epsilon} r_{n}\right)
$$

and hence

$$
T^{1-2 \epsilon}\left(r_{n}\right)<r_{n}^{\gamma+\eta_{1}}
$$$$
\left[\eta_{1}>0\right]
$$

provided $n$ is large enough.

Now (2.1) and (5.1) imply

$$
\gamma \leqq \beta \text {. }
$$

In view of (5.4), (5.5), (5.6), and (5.17), it is obvious that (5.16) cannot hold for large values of $n$. This contradiction proves the lemma.

6. Proof of Theorem 1. We assume that those roots of (3), (4), and (5) which do not lie on the radii (1) are of modulus less than one. This is obviously no restriction because we may, if necessary, consider the function

$$
f^{*}(z)=\frac{1}{\lambda^{l}} f(\lambda z)
$$

instead of $f(z)\left(^{5}\right)$. It is also no restriction to assume, as we shall indeed, that $f(z)$ is not a rational function.

The three functions

$$
f, \quad \frac{1}{f}, \quad \frac{1}{f^{(l)}-1}
$$

(5) If we assume that $f(z)$ has an essential singularity at infinity, but is not necessarily meromorphic, the same transformation enables us to enclose, in the open unit disk, all the nonpolar singularities other than $\infty$. The order of the essential singularity and the deficiency of its poles have the same formal definitions in terms of $m(r, f)$ and $N(r, f)$ [now defined for $r>1$ ]. 
have the same order $\left(^{6}\right) \rho$. Let $h(z)$ denote the first of these three functions which has poles of positive deficiency. This function exists, by assumption (6).

If Theorem 1 were not true, the function $h(z)$ would satisfy all the assumptions of Lemma 3 and therefore (5.3) would hold for some $k$.

On the other hand, we may apply Lemma 1 to the function $f(z)$, so that one of the relations (3.1), (3.2), (3.3) would contradict (5.3). Theorem 1 is thus proved.

7. Proof of Theorem 3. We first prove

LEMma 4. Let $P(z)$ be an entire function of finite order. Assume that its zeros are either real or else lie in the circle $|z| \leqq 1 / 2$.

Consider

$$
H_{1}(w)=\frac{P^{\prime}(i \phi(w))}{P(i \phi(w))}, \quad H_{2}=\frac{P^{\prime}(-i \phi(w))}{P(-i \phi(w))},
$$

where the function $z=\phi(w)$, defined in $\S 2$, maps the circle $|w|<1$ onto the region

$$
\operatorname{Re} z>0,|z|>1 \text {. }
$$

Then, as $t \rightarrow 1(0<t<1)$,

$$
m\left(t, H_{1}(w)\right) \text { and } m\left(t, H_{2}(w)\right)
$$

remain bounded.

Proof. In the region $|z|>1 / 2$, the zeros of $P(z)$ are real and form a sequence $\left\{a_{v}\right\}_{v=1}^{\infty}$ (as usual, multiple zeros are repeated a suitable number of times).

By assumption, $P(z)$ is of the form

$$
P(z)=U(z) \exp (V(z)) \prod_{v=1}^{\infty}\left(1-\frac{z}{a_{v}}\right) \exp \left(\frac{z}{a_{v}}+\cdots+\frac{z^{p}}{p a_{v}^{p}}\right),
$$

where $U(z)$ and $V(z)$ are polynomials, and the $a$ 's such that $\sum\left|a_{v}\right|^{-p-1}$ $<+\infty$. It is no restriction to assume that $p$ is even. If $p$ were odd, we would write

$$
\begin{aligned}
P(z)=U(z) & \exp \left(V(z)-z^{p+1} \sum_{v=1}^{\infty} \frac{1}{(p+1) a_{v}^{p+1}}\right) \\
& \cdot \prod_{v=1}^{\infty}\left(1-\frac{z}{a_{v}}\right) \exp \left(\frac{z}{a_{v}}+\cdots+\frac{z^{p+1}}{(p+1) a_{v}^{p+1}}\right),
\end{aligned}
$$

$\left.{ }^{6}\right)$ In the case of a meromorphic function, the invariance of the order under differentiation has been proved by Valiron [9, p. 129]. His proof and also Nevanlinna's first fundamental theorem are easy to extend to the more general class of functions considered in footnote 5 . Hence, if $f(z)$ belongs to this class, the essential singularities at infinity of the three functions (6.1) have the same order. 
so that a change of notation would yield (7.1) with an even value of $p$.

Now

$$
\frac{P^{\prime}(z)}{P(z)}=\frac{U(z)}{U(z)}+V^{\prime}(z)+z^{p} h(z)
$$

where

$$
V^{\prime}(z)=\sum_{\mu=0}^{l} c_{\mu} z^{\mu}, \quad h(z)=\sum_{v=1}^{\infty} \frac{1}{a_{v}^{p}\left(z-a_{v}\right)} .
$$

Hence

$$
\begin{aligned}
m\left(t, H_{1}(w)\right) \leqq & m\left(t, \frac{U^{\prime}(i \phi(w))}{U(i \phi(w))}\right)+\sum_{\mu=0}^{l} m\left(t, c_{\mu}\{i \phi(w)\}^{\mu}\right) \\
& +m\left(t,\{i \phi(w)\}^{p}\right)+m(t, h(i \phi(w)))+\log (l+3) .
\end{aligned}
$$

For $|w|<1$,

(i) the function

$$
\frac{U^{\prime}(i \phi(w))}{U(i \phi(w))}
$$

is bounded,

(ii) $\operatorname{Re} \phi(w)>0$,

(iii) $\operatorname{Im} h(i \phi(w))<0$,

so that a result of Nevanlinna [5, p. 155] implies the boundedness of each term of the right-hand side of (7.2). The same arguments show that $m\left(t, H_{2}(w)\right)$ remains bounded, and our lemma is proved. Theorem 3 now follows from a conflict between Lemma 1 and Lemma 3.

Consider the function

$$
f(z)=\frac{g(z)}{g^{\prime}(z)}=\frac{P(z)}{P^{\prime}(z)+P(z) Q^{\prime}(z)} .
$$

By assumption all but a finite number of its zeros and poles are real. Furthermore,

$$
f^{\prime}(z)=1
$$

only if

$$
g(z) g^{\prime \prime}(z)=0 .
$$

Hence the roots of the three equations

$$
f(z)=0, \quad f(z)=\infty, \quad f^{\prime}(z)=1
$$

are distributed on the real axis. 
It is obviously no restriction to assume that those roots of the equations (7.3) which are not real belong to the circle $|z| \leqq 1 / 2$. Applying Lemma 1 to the function $f$, we obtain

$$
m\left(t, \frac{P^{\prime}(i \phi(w))}{P(i \phi(w))}+Q^{\prime}(i \phi(w))\right)=O\left(\log \frac{1}{1-t}\right) \quad[t \rightarrow 1,0<t<1],
$$

and a similar relation where $i$ is replaced by $-i$.

In view of Lemma 4, (7.4) yields

$$
m\left(t, Q^{\prime}( \pm i \phi(w))\right)=O\left(\log \frac{1}{1-t}\right) .
$$

On the other hand, the function $Q^{\prime}$ has no poles so that, if its order exceeds 1 , Lemma 3 implies either

$$
\limsup _{t \rightarrow 1} \frac{m\left(t, Q^{\prime}(i \phi(w))\right)}{\log (1 /(1-t))}=+\infty,
$$

or else a similar relation where $i$ is replaced by $-i$. As this contradicts (7.5), the order of $Q^{\prime}$ cannot exceed 1 and Theorem 3 is proved.

8. An elementary application. We first determine all nonreal entire functions of the class $\mathcal{A}$.

Let $f(z)$ be such a function. In view of our corollary,

$$
\begin{aligned}
f(z) & =e^{i(\lambda z+\mu)} g(z), \\
f(z)-1 & =e^{i\left(\lambda_{1} z+\mu_{1}\right)} g_{1}(z),
\end{aligned}
$$

where the constants $\lambda, \lambda_{1}, \mu, \mu_{1}$ are real and the entire functions $g(z)$ and $g_{1}(z)$ are real for real values of $z$. Hence the functions $g$ and $g_{1}$ must satisfy the identities

$$
\begin{aligned}
g(z) \cos (\lambda z+\mu)-1 & =g_{1}(z) \cos \left(\lambda_{1} z+\mu_{1}\right), \\
g(z) \sin (\lambda z+\mu) & =g_{1}(z) \sin \left(\lambda_{1} z+\mu_{1}\right) .
\end{aligned}
$$

Proposition A now follows from a very elementary discussion of this system. This discussion will be omitted.

The proof of proposition B is an easy consequence of the following

Lemma 5. Let $f(z)$ be a real entire function of the class $\mathcal{A}$. Consider an interval I defined by

$$
I: x_{1}<x<x_{2}
$$

such that

(i) $f\left(x_{1}\right)=f\left(x_{2}\right)=1$,

(ii) $f(x) \neq 1$ if $x \in I$.

Then the equation 


$$
f(z)=h
$$$$
(0<h<1)
$$

has no complex zeros in the strip

$$
x_{1} \leqq \operatorname{Re} z \leqq x_{2} .
$$

Proof. Consider the rectangular contour $R(\lambda)$ with vertices at

$$
x_{1} \pm i \lambda, \quad x_{2} \pm i \lambda
$$

It is easily verified that, if $\lambda$ is large enough,

$$
|f(z)| \geqq 1,
$$

at all points of $R(\lambda)$. Hence, by Rouché's Theorem, $f(z)$ and $f(z)-h$ have the same number of zeros in the strip (8.2).

We next deduce from a theorem of Laguerre [2, p. 32] that, in the interval $I, f(z)$ has either

(i) two simple zeros, or

(ii) one double zero, or

(iii) no zeros.

Hence, in the cases (i) and (ii), a simple continuity argument shows that the equation (8.1) has at least two real simple zeros in the interval $I$. The lemma is now obvious.

If the equation

$$
f(z)=1
$$

has an infinity of positive roots and an infinity of negative roots, $\mathrm{B}$ follows immediately from Lemma 5 . In all other cases $\left({ }^{7}\right)$ our arguments require minor modifications which will be left to the reader.

In order to prove $C$, we consider the function

$$
F(z)=\frac{f(z)-h_{1}}{h_{2}-h_{1}},
$$

which belongs to the class $\mathcal{A}$. This function is certainly not bounded on the real axis and hence does not reduce to one of the exceptional forms mentioned in proposition $\mathrm{A}$. by

Hence $F(z)$ is real for all real values of $z$ and the sequence $\left\{h_{0}^{*}\right\}_{0=1}^{\infty}$ defined

$$
h_{v}^{*}=\frac{h_{v}-h_{1}}{h_{2}-h_{1}}
$$

(7) If $f(z)-1$ has only a finite number of positive [or negative] zeros, there are also only a finite number of positive [or negative] zeros of $f(z)$. In view of our corollary, $f(z)$ is of order not greater than $1 / 2$. 
is real and unbounded. Considering, if necessary, a subsequence of $\left\{h_{0}^{*}\right\}$ instead of the complete sequence, and $-F(z)$ instead of $F(z)$, we may assume

$$
h_{v}^{*}>0 \quad(v=2,3, \cdots) ; \quad h_{v}^{*} \rightarrow+\infty .
$$

Then, by $B$, all the roots of

$$
F(z)=h
$$

are real, for all real non-negative values of $h$.

Hence all the zeros and ones of the entire function

$$
\Lambda(z)=\cos (F(z))^{1 / 2}
$$

are real. By our corollary, the order of $\Lambda(z)$ does not exceed one so that, in view of a theorem of Pólya [7, p. 12], $F(z)$ is a polynomial. It is now trivial to verify that the degree of $F(z)$ does not exceed 2 .

\section{REFERENCES}

1. O. Blumenthal, Principes de la thêrie des fonctions entières d'ordre infini, Paris, 1910.

2. E. Borel, Leģons sur les fonctions entières, 2d ed., Paris, 1921.

3. H. Milloux, Quelques propriettes des fonctions entières d'ordre infini, Ann. Ecole Norm. vol. 49 (1932) pp. 311-350.

4. - Les fonctions meromorphes et leuirs dérivees, Paris, 1940.

5. R. Nevanlinna, Le theorème de Picard-Borel et la theorie des fonctions meromorphes, Paris, 1929.

6. G. P6lya, Über die Nullstellen sukzessiver Derivierten, Math. Zeit. vol. 12 (1922) pp. 3660.

7. - On an integral function of an integral function, J. London Math. Soc. vol. 1 (1926) pp. 12-15.

8. W. Saxer, Über die Picardschen Ausnahmewerte sukzessiver Derivierten, Math. Zeit. vol. 17 (1923) pp. 206-227.

9. G. Valiron, Sur la distribution des valeurs des fonctions merromorphes, Acta Math. vol. 47 (1926) pp. 117-142.

10. - Sur les valeurs exceptionnelles des fonctions meromorphes et de leurs derivees, Paris, 1937.

SYRACUSE UNIVERSITY, Syracuse, N. Y. 